\title{
Psoriasis: Pluspunkte für Antikörper
}

\section{Die Therapie von Psoriasispatienten mit dem monoklonalen Antikörper Brodalumab hat sich in bisherigen Studien als wirksam erwiesen. Jetzt liegen die Ergebnisse der Phase-III-Studie AMAGINE-1 vor.}

ei AMAGINE-1 handelt es sich um eine prospektive placebokontrollierte Studie, an der 661 Patienten mit mittelschwerer bis schwerer Psoriasis teilnahmen. Sie waren zwischen 18 und 75 Jahre alt und hatten zu Studienbeginn seit mindestens einem halben Jahr eine stabile Erkrankung. Mindestens $10 \%$ der Körperoberfläche waren von den Hautveränderungen betroffen. Der PASI (Psoriasis Area and Severity Index) lag bei mindestens 12, der PGA (Physician's Global Assessment) bei mindestens 3. Ziel der Studie war unter anderem, Wirksamkeit und Sicherheit des Präparates zu überprüfen.

220 Patienten erhielten ein Scheinpräparat, 219 Patienten den Antikörper in einer Dosis von $140 \mathrm{mg}$ und 222 Patienten in einer Dosis von $210 \mathrm{mg}$, und zwar alle zwei Wochen subkutan. In der Absetzphase der Studie erhielten Patienten, die ursprünglich Brodalumab in niedriger oder hoher Dosierung erhalten hatten und bei denen der PGA bei 0 oder 1 lag, nach zwölf Wochen den Antikörper oder Placebo. Ab der Woche 16 erhielten Patienten mit Wiederaufflammen der Erkrankung $($ PGA $\geq 3$ ) ihre Induktionsdosis. Wenn nach zwölf Wochen erneuter Therapie kein Ansprechen zu verzeichnen war, wurde den Patienten alle zwei Wochen $210 \mathrm{mg}$ Brodalumab subkutan injiziert. Geprüft wurde zum einen der Anteil der Patienten, bei denen sich eine mindestens 75\%ige Linderung der Symptome (PASI 75) verzeichnen lässt, sowie der PGA-Erfolg zwölf Wochen nach Studienbeginn. Zum anderen wurde der anhaltende Therapieeffekt nach 52 Wochen anhand des PGA bestimmt.

Durch die Behandlung wurde nach zwölf Wochen der PASI 75 bei $60 \%$ der Patienten mit 140 mg und bei $83 \%$ der Patienten mit 210 mg Brodalumab erzielt. In der Kontrollgruppe waren es nur $3 \%$. Ein PGA 0 oder 1 wurde bei $54 \%$ und $76 \%$ erreicht, unter Placebo bei $1 \%$ der Patienten. Bei mehr als zwei Drittel der Patienten, die nach zwölf Wochen eine Linderung der Symptome hatten, waren die von Psoriasis betroffenen Hautareale nach einem Jahr verschwunden.

Die häufigsten unerwünschten Wirkungen ( $\geq 5 \%$ ) waren Nasopharyngitis, Infektionen der oberen Atemwege sowie Kopfschmerzen. Mindestens eine dieser Nebenwirkungen hatten $59 \%$ der Patienten bei einer Dosis von $210 \mathrm{mg}$, 58\% bei 140 mg und $51 \%$ der Patienten in der Placebogruppe.

Fazit: Durch die Behandlung von Psoriasispatienten mit Brodalumab gelingt es, bei vielen Patienten die Beschwerden drastisch zu lindern, auch langfristig. Das Nebenwirkungsprofil ist nach Ansicht der Studienärzte bei Patienten mit mittelschwerer oder schwerer Erkrankung vertretbar.

\section{Hier steht eine Anzeige.}

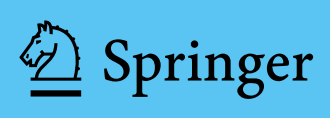

Papp KA et al. A prospective phase 3, randomized, double-blind, placebocontrolled study of brodalumab in patients with moderate-to-severe plaque psoriasis. Br J Dermatol 2016; doi: 10.1111/bjd.14493 